\section{6-2.4 EARLY LIFE EXPOSURES AND THE DEVELOPMENT OF TESTICULAR CANCER IN NORTHERN IRELAND: A RETROSPECTIVE BIRTH-COHORT STUDY}

doi:10.1136/jech.2011.142976b.74

${ }^{1} \mathrm{~S}$ Alam, ${ }^{* 1} \mathrm{M}$ Cantwell, ${ }^{1} \mathrm{C}$ Cardwell, ${ }^{2} \mathrm{~A}$ Gavin, ${ }^{1} \mathrm{~L}$ Murray. ${ }^{1}$ Queen's University, Belfast, UK; ${ }^{2}$ Northern Ireland Cancer Registry, Belfast, UK

Objectives To investigate perinatal risk factors for testicular cancer in a Northern Ireland population cohort.

Methods Perinatal data have been routinely recorded in Northern Ireland for all births in the period 1971-1986 ( $n=447,663)$. Testicular cancer status was ascertained in this cohort by identifying 249 individuals diagnosed from 1971 to 2008 and date of birth in the period 1971-1986.

Results Increased testicular cancer risk was associated with higher maternal age ( $>35$ years), lower birth weight $(<2500 \mathrm{~g})$, caesarean delivery, decreased birth order and lower socio-economic status. Following multivariable analyses, the association between higher maternal age and testicuar cancer risk remained significant (OR 1.55; 95\% CI 1.07 to 2.25). Caesarean delivery was also associated with a significant increase in the risk of testicular cancer (OR 1.65; $95 \%$ CI 1.11 to 2.44 ). Increasing birth order was associated with a significant decrease in the testicular cancer risk (OR 0.59; 95\% CI 0.41 to 0.83 ) comparing birth order three or more with the firstborn. Conclusion These findings demonstrate that maternal age at delivery, birth order and mode of delivery are significantly associated with testicular cancer risk. These associations may be due to earlier exposure to infectious agents or increased immune modulation.

\section{6-2.5 ALCOHOL AND PROSTATE CANCER RISK: A MENDELIAN RANDOMISATION APPROACH}

doi:10.1136/jech.2011.142976b.75

1,2 L Zuccolo, ${ }^{* 1,2}$ S Lewis, ${ }^{3} \mathrm{~F}$ Hamdy, ${ }^{4} \mathrm{D}$ Neal, ${ }^{2} \mathrm{~J}$ Donovan, ${ }^{1,2} \mathrm{G}$ Davey Smith. ${ }^{1} \mathrm{MRC}$ Centre for Causal Analyses in Translational Epidemiology, University of Bristol, Bristol, UK; ${ }^{2}$ School of Social and Community Medicine, University of Bristol, Bristol, UK; ${ }^{3}$ Nuffield Department of Surgery, University of Oxford, Oxford, UK; ${ }^{4}$ Department of Oncology and Department of Surgery, University of Cambridge, Cambridge, UK

Introduction Despite alcohol being an established carcinogen and recent prospective studies showing increased prostate cancer risk among heavy drinkers, alcohol is not an established risk factor for prostate cancer.

We aimed to investigate the causal role of alcohol on prostate cancer through conventional observational epidemiology techniques and Mendelian randomisation, by using genetic variants influencing the propensity to drink or modifying the physiological response to alcohol.

Methods A case-control study was nested in the case identification phase of a large British population-based RCT for treatment of localised prostate cancer (Protec T). 2400 prostate-specific antigen detected prostate cancer cases of white ethnicity and 12700 controls matched on age and general practice provided data on alcohol consumption. Eighteen SNPs in alcohol-metabolising genes (ADH's, ALDH2) were genotyped in a sub-sample of cases and controls.

Results There was some evidence of a modest decrease in low Gleason-grade (RR 0.96; 95\% CI 0.93 to 0.99 ) and increase in high-grade (RR 1.04; 95\% CI 0.99 to 1.08 ; p difference=0.004) prostate cancer per 10 alcohol units/week increase in consumption, not explained by current BMI, blood pressure, co-morbidities, or reverse causation.

Results from genetic association analyses including an interaction between an $\mathrm{ADH} 1 \mathrm{~B}$ functional variant and alcohol consumption suggested that alcohol causally increases prostate cancer risk. However, this study was underpowered to detect a difference between results from instrumental variable analyses and conventional observational epidemiology models.

Conclusion These results support small increases in high-grade prostate cancer risk caused by heavy alcohol drinking. The required independent replication in populations with incident (non prostate-specific antigen-detected) prostate cancer is currently under-way.

\section{6-2.6 BLADDER CANCER SURVIVAL DISPARITIES IN THE UNITED STATES: RESULTS FROM SEER-MEDICARE}

doi:10.1136/jech.2011.142976b.76

${ }^{1} \mathrm{G}$ Datta, ${ }^{2} \mathrm{P}$ Grosclaude, ${ }^{3} \mathrm{~K}$ Kawachi, ${ }^{4} \mathrm{~B}$ Neville, ${ }^{5} \mathrm{~N}$ Datta, ${ }^{6} \mathrm{C}$ Earle. ${ }^{1}$ University of Montreal, Montreal, Quebec, Canada; ${ }^{2}$ INSERM U558, Toulouse, France; ${ }^{3}$ Harvard School of Public Health, Boston, Massachusetts, USA; ${ }^{4}$ Dana-Farber Cancer Institute, Boston, Massachusetts, USA; ${ }^{5}$ University of California, Los Angeles, Los Angeles, California, USA; ${ }^{6}$ University of Tornoto, Toronto, Ontario, Canada

Introduction Black patients have lower bladder cancer survival rates than White patients, but previous studies have not been able to explain this difference. Recent work has found that racial disparities in bladder cancer survival persist after adjusting for sex, age, and tumour characteristics. The objective of this study was to assess the association of insurance status, comorbidities, marital status, the receipt of radical cystectomy and being qualified for federal assistance (a marker for low individual-level SES) with disparities in bladder cancer survival.

Methods We identified 15666 (592 Black and 15074 White) bladder cancer cases diagnosed between 1992 and 1999 (follow-up through 2003) from the SEER-Medicare database, and constructed relative survival models to assess 5-year survival disparities.

Results The relative survival ratios (RSR) for Black patients vs White patients were as follows: unadjusted, RSR 2.22 (95\% CI 1.90 to 2.59), adjusting for year of diagnosis, registry, age, sex, stage, and grade, RSR 1.54 (95\% CI 1.32 to 1.81); additionally adjusting for comorbidity score, marital status, and receipt of radical cystectomy, RSR 1.45 (95\% CI 1.25 to 1.70); additionally adjusting for SES, RSR 1.26 (95\% CI 1.08 to 1.48). Lower comorbidity score, being married, higher SES and receipt of radical cystectomy were independently associated with increased bladder cancer survival.

Conclusions Racial disparities persist after adjusting for comorbidity score, marital status, receipt of radical cystectomy and SES in this insured population. As the SES variable used in this study does not capture the diversity across the SES gradient and it is strongly associated with race, further studies investigating the influence of SES and its correlates may provide further explication of this disparity.

\subsection{EPIDEMIOLOGY AND POLICY}

\section{Chair: Dr. Gerry McCartney, UK 06-3.1 MAPPING INJUSTICE, VISUALISING EOUITY: A JOINT PRESENTATION ON SITUATING AND TACKLING HEALTH INEQUITIES}

doi:10.1136/jech.2011.142976b.77

${ }^{1}$ N Krieger, ${ }^{*}{ }^{2} \mathrm{D}$ Dorling, ${ }^{*} \mathrm{G}$ McCartney. ${ }^{*}$ Department of Society, Human Development, and Health, Harvard School of Public Health, Boston, Massachusetts, USA

${ }^{2}$ University of Sheffield, Sheffield, UK; ${ }^{3}$ NHS Health Scotland, Glasgow, UK

Introduction Epidemiological theory matters for seeing, analysing, depicting and tackling population distributions of health and health 
inequities. The contrasts between the dominant (biomedical and lifestyle) and alternative (socio-political, psychosocial and ecosocial) epidemiological theories are present in the imagery used to illustrate population health distributions. This symposium will provoke deeper thinking of how health distributions are created by exploring the interplay between theory and imagery.

Methods The competing theories to explain health distributions will be presented and critiqued in relation to case studies across the world. The theoretical underpinnings to imagery in medicine and politics will then be exposed with a particular focus on the iconography used in explaining health inequalities. The Worldmapper resource and Unnatural Causes video will then be showcased prior to a group discussion on the implications of the presented material.

Results Competing epidemiological theories have their own imagery and iconography which reinforce their value base. Awareness of the underlying theory should provoke epidemiologists to consider which images and icons they utilise when attempting to explain their findings.

Conclusion Health inequity explanations can be lost in translation if inappropriate images and icons are utilised. Explanations, imagery and icons should be consistent if the causes of inequities are to be widely understood. 